\title{
Aspectos termodinámicos de la miscibilidad parcial entre el $n$-octanol y el agua
}

\author{
Carolina P. Mora, Hernán R. Lozano, Fleming Martínez ${ }^{1 *}$ \\ ${ }^{1}$ Departamento de Farmacia, Universidad Nacional de Colombia, Bogotá D.C., Colombia
}

*Correspondence:

F. Martinez

Universidad Nacional de Colombia

Departamento de Farmacia

A.A. 14490

Bogotá - D.C. - Colombia

E-mail:fmartinezn@unal.edu.co
En este trabajo se realizó el tratamiento termodinámico de los datos en fracción molar del equilibrio líquido-líquido entre noctanol y agua en función de la temperatura, utilizando el método de van't Hoff (In $\mathrm{S}$ en función de $\mathrm{T}^{-1}$ ) y el planteado por Grant et al. (ln $\mathrm{S}$ en función de $\mathrm{T}^{-1}$ ) y ln $\mathrm{T}$ : para las dos escalas de concentración. Se encontró un comportamiento no lineal para las dos fases mutuamente saturadas en el tratamiento de van't Hoff, por lo que se utilizó un modelo de regresión parabólico, que fue derivado para resolver el cambio entálpico de solución, obteniendo procesos endotérmicos a todas las temperaturas estudiadas. En los dos sistemas los cambios de energía libre fueron positivos mientras que los cambios entrópicos fueron negativos indicando algún grado de organización en las soluciones saturadas, que en el caso del agua saturada de n-octanol podría explicarse por la hidratación hidrofóbica en torno a las cadenas octílicas y en el caso del noctanol saturado de agua podría deberse como ha sido planteado en la literatura a la organización de las moléculas del n-octanol entorno a las moléculas de agua mediante la formación de enlaces de hidrógeno. Los valores termodinámicos calculados fueron comparados con los presentados por otros autores, obtenidos por calorimetría y también, mediante evaluación de constantes de equilibro en función de la temperatura.
Unitérminos

- Sistema n-octanol-agua

- Miscibilidad parcial

- Termodinámica de soluciones

\section{INTRODUCCIÓN}

El sistema de reparto $n$-octanol-agua ha sido considerado por mucho tiempo como el más relevante en el planteamiento de correlaciones cuantitativas entre la estructura molecular y la actividad biológica (QSAR) de numerosas series de compuestos. Aunque en estudios recientes se han utilizado sistemas más complejos que simulan mejor el ambiente biológico celular, tales como las micelas y las vesículas liposomales, este sistema de reparto líquido-líquido continúa siendo ampliamente utilizado en estudios farmacológicos, toxicológicos y ambientales (Hansch, Leo, 1995; Sangster, 1997). La principal razón de la utilización del $n$-octanol en estudios QSAR radica en su adecuado balance 
hidrófilo-lipófilo, su capacidad de formación de enlaces de hidrógeno y su contenido relativamente alto de agua de saturación, que simulan adecuadamente el comportamiento semipolar de las membranas celulares, las cuales son un paso obligado durante el proceso de absorción de xenobióticos (Leo et al., 1971; Martínez et al., 2000).

Algunas consideraciones termodinámicas y teóricas indican que el n-octanol saturado de agua posee una estructura microheterogénea, mientras que el agua saturada de n-octanol posee una estructura ligeramente más asociada que el agua pura (DeBolt y Kollman, 1995; Martínez et al., 2001a). También Franks et al. (1993) mediante análisis de difracción de rayos $\mathrm{X}$ han demostrado la existencia de una organización molecular en forma de estructura microheterogénea en el n-octanol líquido como solvente puro y como solvente orgánico saturado de agua, basada en el efecto cooperativo de los enlaces de hidrógeno. Por estas razones se ha encontrado que las correlaciones cuantitativas entre la actividad biológica y el coeficiente de reparto en este sistema son mucho más adecuadas que las obtenidas en otros sistemas tales como hidrocarburo-agua (Ávila y Martínez, 2003).

Si bien desde hace bastante tiempo se utiliza la calorimetría para determinar el cambio entálpico de transferencia de fármacos desde el agua hasta el $n$-octanol, aún hoy en día se sigue utilizando el método de van't Hoff (basado en la variación del coeficiente de reparto con la temperatura) para hallar esta función termodinámica. Un cuestionamiento realizado al respecto ha sido el referido a la variación del equilibrio líquido-líquido debido a la variación de la solubilidad con la temperatura en este sistema de reparto, que podría afectar los valores de las funciones termodinámicas calculadas para los procesos de transferencia estudiados por este método. Por esta razón, diversos autores han estudiado la saturación mutua en función de la temperatura para este par de solventes, estos valores han sido compilados por Sangster (1997). Un trabajo sistemático y relativamente reciente, ha sido presentado por Dallos y Liszi (1995) en el cual se estudió la saturación mutua en fracción molar desde 15 hasta $50^{\circ} \mathrm{C}$ presentando adicionalmente la densidad de cada una de las fases líquidas.

La metodología seguida por estos investigadores consistió básicamente en la purificación de los dos solventes por destilación descartando las cabezas y las colas obtenidas en los procesos, saturación mutua de los dos solventes mediante agitación continua durante al menos siete horas a las diferentes temperaturas de estudio, posteriormente la separación de las dos fases y determinación de las respectivas densidades utilizando un densímetro digital Anton Paar 602, y finalmente, la cuantificación del soluto respectivo, n-octanol presente en el agua mediante cromatografía gas-líquido, y agua presente en el n-octanol mediante el método de KarlFischer.

Este trabajo es el que ha sido adoptado como base para el desarrollo de los diferentes trabajos de transferencia desde el agua hasta el n-octanol que han sido adelantados en nuestro grupo de investigación (Martínez et al., 2001b; Martínez y Gómez, 2002; Ávila y Martínez, 2003; Baena et al., 2004).

Como un aporte a la generación de información fisicoquímica relativa al sistema n-octanol-agua, en el presente trabajo se procesaron termodinámicamente los datos de equilibrio presentados por Dallos y Liszi (1995) utilizando el clásico método de van’t Hoff (Martínez et al., 2000) y el expuesto por Grant et al. (1984), los cuales están basados en la variación de la solubilidad con la temperatura, para hallar las respectivas funciones termodinámicas, energía libre, entalpía y entropía de solución, interpretando los resultados en términos de interacciones solutosolvente.

\section{Aspectos termodinámicos clásicos de solución}

La elaboración de gráficos ponderados del logaritmo de la solubilidad en función de la temperatura absoluta recíproca permite hallar el cambio entálpico aparente de solución $\left(\Delta H^{\text {app }}{ }_{\text {soln }}\right)$ a partir de la ecuación de van't Hoff (Ec. 1):

$$
\left(\frac{\partial \ln X_{2}}{\partial(1 / T)}\right)_{P}=-\frac{\Delta H_{\text {soln }}^{\mathrm{app}}}{R}
$$

Donde $R$ es la constante universal de los gases (8.314 $\mathrm{J} \mathrm{mol}^{-1} \mathrm{~K}^{-1}$ ). Sin embargo en tratamientos más recientes se han introducido correcciones a la expresión anterior para disminuir la propagación de errores y por lo tanto diferenciar entre efectos químicos propiamente dichos y aquellos debidos únicamente al tratamiento estadístico utilizado en la regresión, lo que ha llevado a utilizar preferentemente la denominada temperatura armónica media $\left(T_{h m}\right)$, la cual generalmente corresponde a la temperatura absoluta promedio obtenida entre la más alta y la más baja estudiadas (Krug et al., 1976). La expresión corregida más utilizada en la literatura (Bustamante et al., 1998) es la siguiente:

$$
\left(\frac{\partial \ln X_{2}}{\partial\left(1 / T-1 / T_{h m}\right)}\right)_{P}=-\frac{\Delta H_{s o l n}^{\mathrm{app}}}{R}
$$


Cuando no se obtienen comportamientos lineales en la regresión, estos resultados indican que $\Delta H^{\text {app }}$ cambia con la temperatura en el intervalo estudiado, lo cual a su vez, en primer lugar lleva a plantear modelos polinómicos de regresión de orden dos (Ec. 3: modelos parabólicos):

$$
y=a+b x+c x^{2}
$$

En los cuales, $y=\ln X_{2}$ y $x=T^{-1}$. Estas ecuaciones son derivadas y resueltas punto a punto para hallar $\Delta H_{\text {soln }}^{\text {app }}$ a cada temperatura mediante la siguiente expresión:

$$
\Delta H_{\mathrm{soln}}^{\mathrm{app}}=-R \frac{d y}{d x}=-R(b+2 c x)
$$

Una segunda forma de procesar datos de solubilidad en función de la temperatura cuando estos no son lineales en la relación de van't Hoff es mediante la siguiente ecuación presentada por Grant et al. (1984), que también es conocida como Ecuación de Kirchhoff (Van Ness y Abbott, 1982):

$$
\ln X_{2}=-a \frac{1}{T}+b \ln T+c
$$

A partir de la cual, la entalpía aparente de solución se calcula utilizando los coeficientes $a$ y $b$ de la Ec. 5 mediante la expresión:

$$
\Delta H_{\text {soln }}^{\mathrm{app}}=a R+b R T
$$

La Ec. 6 puede utilizarse para calcular $\Delta H^{\text {app }}{ }_{\text {soln }}$ a cualquier temperatura dentro del intervalo estudiado, sin embargo los resultados son más confiables cuando esta propiedad se calcula a la temperatura armónica.

En las ecuaciones anteriores se ha utilizado la concentración del soluto en la solución $\left(X_{2}\right)$ en lugar de la actividad termodinámica correspondiente del soluto $\left(a_{2}\right)$ por lo que en tratamientos más elaborados se han utilizado correcciones para hacer más coincidentes los valores de entalpía de van't Hoff con los obtenidos por técnicas calorimétricas, uno de estos tratamientos es el planteado en la siguiente ecuación (Hollenbeck, 1980; Bustamante et al., 1998):

$$
\Delta H_{\mathrm{soln}}=\Delta H_{\mathrm{soln}}^{\mathrm{app}}\left(\frac{\partial \ln a_{2}}{\partial \ln X_{2}}\right)_{T, P}
$$

Donde, el segundo término de la derecha represen- ta la desviación en la entalpía respecto al valor real debido a posibles variaciones de la actividad termodinámica del soluto con la concentración del mismo en las soluciones, y se puede calcular a partir de la Ec. 8 (Manzo y Ahumada, 1990; Bustamante et al., 1998):

$$
\left(\frac{\partial \ln a_{2}}{\partial \ln X_{2}}\right)_{T, P}=1-\frac{2 \phi_{2}}{X_{1}} \ln \left(\frac{a_{2}^{\text {sat }}}{X_{2}^{\mathrm{sat}}}\right)
$$

En la cual, los superíndices "sat" indican la condición de saturación y $\phi_{2}$ es la fracción volumétrica del soluto en la solución la cual puede hallarse utilizando el concepto de volumen de desplazamiento $\left(D_{2}\right)$, el cual es una adaptación práctica del concepto termodinámico de volumen molar aparente (Pérez et al., 2003). La expresión más utilizada para calcular $D_{2}$ es:

$$
D_{2}=\frac{m_{2}+m_{1}\left(1-D_{1} \rho\right)}{m_{2} \rho}
$$

En la cual, $m_{2}$ y $m_{1}$ son las masas (g) del soluto y el solvente, $D_{1}$ es el desplazamiento del solvente (densidad recíproca, $\mathrm{cm}^{3} \mathrm{~g}^{-1}$ ) y $\rho$ es la densidad de la solución $\left(\mathrm{g} \mathrm{cm}^{-3}\right)$ (Pérez et al., 2003).

Puesto que los solutos estudiados en este trabajo son líquidos, lo que conduce a que la actividad termodinámica de estos en la saturación se considere unitaria (Yalkowsky, 1999), propiedad que es coincidente con la solubilidad ideal, entonces se plantea la Ec. 10 para hacer utilizable directamente la Ec. 8 mediante la Ec. 11.

$$
\left(\frac{a_{2}^{\text {sat }}}{X_{2}^{\text {sat }}}\right)=\left(\frac{1}{X_{2}^{\text {sat }}}\right)
$$

$$
\left(\frac{\partial \ln a_{2}}{\partial \ln X_{2}}\right)_{T, P}=1-\frac{2 \phi_{2}}{X_{1}} \ln \left(\frac{1}{X_{2}^{\text {sat }}}\right)
$$

El cambio de energía libre estándar para el proceso de solución $\left(\Delta G_{\text {soln }}^{\text {app }}\right)$ se ha calculado tradicionalmente como:

$$
\Delta G_{\text {soln }}^{\text {app }}=-R T_{\mathrm{hm}} \ln X_{2}
$$

El cual puede también ser normalizado multiplicando por el factor $\left(\partial \ln a_{2} / \partial \ln X_{2}\right)_{T, P}$ para expresar la función en términos de actividad termodinámica en lugar de concentración.

En cambio entrópico estándar aparente de solución $\left(\Delta S_{\text {soln }}^{\text {app }}\right)$ se obtiene directamente a partir de los valores aparentes de entalpía y energía libre utilizando la expresión: 


$$
\Delta S_{\mathrm{soln}}^{\mathrm{app}}=\frac{\left(\Delta H_{\mathrm{soln}}^{\mathrm{app}}-\Delta G_{\mathrm{soln}}^{\mathrm{app}}\right)}{T_{h m}}
$$

Los cuales son corregidos mediante la Ec. 14 para obtener los valores reales:

$$
\Delta S_{\mathrm{soln}}=\Delta S_{\mathrm{soln}}^{\mathrm{app}}\left(\frac{\partial \ln a_{2}}{\partial \ln X_{2}}\right)_{T, P}
$$

\section{RESULTADOS Y DISCUSIÓN}

En la Tabla I se presentan los resultados de composición del equilibrio líquido-líquido en fracción molar presentados por Dallos y Liszi (1995) junto a las densidades de las fases mutuamente saturadas en función de la temperatura. En esta misma tabla se presentan los valores de solubilidad en la escala de molaridad calculados mediante la siguiente expresión:

$$
C=\frac{1000 \rho X_{2}}{M_{1}\left(1-X_{2}\right)+M_{2} X_{2}}
$$

En la cual, $\rho$ es la densidad de la solución, $X_{2}$ es la solubilidad del soluto en fracción molar, y $M_{1}$ y $M_{2}$ son las masas molares del solvente y del soluto según el caso. Las masas molares de los dos solventes son $18.02 \mathrm{~g} \mathrm{~mol}^{-1} \mathrm{y}$ $130.23 \mathrm{~g} \mathrm{~mol}^{-1}$ para el agua y el $n$-octanol puros, respectivamente (Martin et al., 1993).

Se observa claramente en la Tabla I que la miscibilidad parcial es dependiente de la temperatura. De otra parte, recordando que la solubilidad ideal para un soluto líquido se considera unitaria, entonces el coeficiente de actividad del soluto en la solución $\left(\gamma_{2}\right)$ se calcula como la solubilidad recíproca en la escala de fracción molar. Estos valores también se presentan en la Tabla I para los dos sistemas.

A partir de los valores de $\gamma_{2}$ se puede hacer una primera aproximación a la estimación de las interacciones intermoleculares entre el soluto y el solvente por medio de la Ec. 16 (Kristl y Vesnaver, 1995; Martínez y Gómez, 2001).

$$
\ln \gamma_{2}=\left(\omega_{11}+\omega_{22}-2 \omega_{12}\right) \frac{V_{2} \phi_{1}^{2}}{R T}
$$

En la cual $\omega_{11} \omega_{22}$ y $\omega_{12}$ representan las energías de interacción solvente-solvente, soluto-soluto y solventesoluto respectivamente, $V_{2}$ es el volumen molar del soluto y $\phi_{1}$ es la fracción volumétrica del solvente. Clásicamente se ha considerado que $\omega_{22}$ representa la energía requerida para pasar una molécula de soluto al estado de vapor, mientras que el término $\omega_{11}$ representa la energía requerida para separar algunas moléculas del solvente de tal forma que se pueda crear una cavidad del tamaño molecular del soluto, y finalmente el término $\omega_{12}$ representa la energía liberada después de que la molécula de soluto se aloja en la cavidad creada en el solvente.

El término compuesto $\left(V_{2} \phi_{1}{ }^{2} / R T\right)$ puede considerarse como constante para el mismo soluto a la misma temperatura y por lo tanto el valor de $\gamma_{2}$ va a depender casi exclusivamente de los términos de interacción $\omega_{11}, \omega_{22}$ y $\omega_{12}$ (Kristl y Vesnaver, 1995; Martínez y Gómez, 2001). En el caso de los dos compuestos estudiados en este trabajo se tiene que las interacciones agua-agua son bastante fuertes debido a la gran capacidad de formación de enlaces de hidrógeno, lo que se manifiesta en la alta polaridad de este compuesto (constante dieléctrica, $\varepsilon=78.5$ y parámetro de solubilidad de Hildebrand, $\delta=23.4 \mathrm{cal}^{1 / 2} \mathrm{~cm}^{-3 / 2}$ a $25.0^{\circ} \mathrm{C}$ (Martin et al., 1993)) mientras que en el caso del $n$-octanol, si bien no es tan

TABLA I - Solubilidad en fracción molar, coeficiente de actividad y solubilidad molar $\left(\mathrm{mol} \mathrm{L}^{-1}\right)$ del $n$-octanol en el agua y del agua en el n-octanol, y densidad $\left(\mathrm{g} \mathrm{cm}^{-3}\right)$ de las soluciones, en función de la temperatura según los datos de Dallos y Liszi (1995).

\begin{tabular}{lcccccccc}
\hline Temp. $/{ }^{\circ} \mathbf{C}$ & \multicolumn{3}{c}{$\boldsymbol{n}$-Octanol en agua } & \multicolumn{4}{c}{ Agua en $\boldsymbol{n}$-octanol } \\
& $\boldsymbol{X}_{\mathrm{ROH}}$ & $\boldsymbol{\gamma}_{\mathrm{ROH}}$ & $\boldsymbol{C}_{\mathrm{ROH}}$ & $\boldsymbol{\rho}$ & $\boldsymbol{X}_{\mathrm{W}}$ & $\boldsymbol{\gamma}_{\mathrm{W}}$ & $\boldsymbol{C}_{\mathrm{W}}$ & $\boldsymbol{\rho}$ \\
\hline 15.0 & 0.000054 & 18519 & 0.00299 & 0.99903 & 0.271 & 3.69 & 2.237 & 0.83591 \\
20.0 & 0.000056 & 17857 & 0.00310 & 0.99814 & 0.273 & 3.66 & 2.249 & 0.83233 \\
25.0 & 0.000059 & 16949 & 0.00326 & 0.99698 & 0.274 & 3.65 & 2.250 & 0.82883 \\
30.0 & 0.000062 & 16129 & 0.00342 & 0.99558 & 0.276 & 3.62 & 2.263 & 0.82542 \\
35.0 & 0.000065 & 15385 & 0.00358 & 0.99396 & 0.278 & 3.60 & 2.274 & 0.82178 \\
40.0 & 0.000069 & 14493 & 0.00380 & 0.99213 & 0.281 & 3.56 & 2.297 & 0.81835 \\
45.0 & 0.000074 & 13514 & 0.00406 & 0.99013 & 0.283 & 3.53 & 2.309 & 0.81483 \\
50.0 & 0.000078 & 12821 & 0.00427 & 0.98793 & 0.286 & 3.50 & 2.331 & 0.81132 \\
\hline
\end{tabular}


polar como el agua, de todas formas las interacciones noctanol-n-octanol son también relativamente fuertes debido a su capacidad de formación de enlaces de hidrógeno y a la estabilización de las cadenas octílicas por fuerzas de dispersión $\left(\varepsilon=10.4\right.$ y $\delta=10.3 \mathrm{cal}^{1 / 2} \mathrm{~cm}^{-3 / 2}$ a $25.0{ }^{\circ} \mathrm{C}$ (Chuman et al., 2002)), lo que conduce a que los términos $w_{11}$ y $w_{22}$ sean relativamente altos en los dos sistemas, según el caso mayor o menor, por lo que el término que más aporta al coeficiente de actividad del soluto es $w_{12}$, esto es el término correspondiente a las interacciones soluto-solvente.

De la Tabla I se tiene que $\gamma_{2}$ para el n-octanol en agua es mayor que 10000 , mientras que $\gamma_{2}$ para el agua en el $n$-octanol es cercano a 3.5, lo que indica que en el primer caso las interacciones soluto-solvente son bastante débiles y por lo tanto los términos que gobiernan son $\omega_{11}$ y $\omega_{22}(\mathrm{el}$ término $\omega_{12}$ posee coeficiente dos veces negativo), mientras que en el segundo caso las interacciones solutosolvente deben ser bastante fuertes lo que lleva a valores $\gamma_{2}$ relativamente bajos. Los posibles tipos específicos de interacciones intermoleculares soluto-soluto, solventesolvente y soluto-solvente presentes en las soluciones

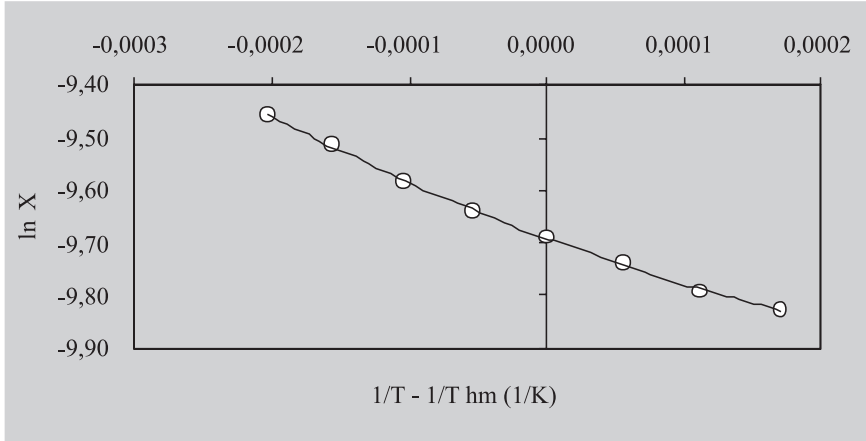

FIGURA 1 - Solubilidad del n-octanol en el agua en fracción molar en función de la temperatura considerando la temperatura armónica (303.15 K). saturadas se expondrán más adelante con base en los respectivos análisis termodinámicos de funciones útiles.

\section{Cálculo de las funciones termodinámicas aparentes de solución}

En las Figuras 1 y 2 se presentan las gráficas de van't Hoff, utilizando la temperatura armónica que en este caso se considera por razones prácticas igual a $30.0^{\circ} \mathrm{C}$, en la escala de fracción molar para el agua saturada de noctanol (solubilidad del n-octanol en el agua) y el n-octanol saturado de agua (solubilidad del agua en el n-octanol). Puede observarse que en los dos casos se obtienen comportamientos no lineales, lo que indica que $\Delta H^{\text {app }}{ }_{\text {soln }}$ cambia con la temperatura en el intervalo estudiado, por lo que los datos se ajustan a modelos parabólicos (Ec. 3), encontrando coeficientes de determinación, superiores a 0.997 (Anexo 1). Estas ecuaciones son derivadas y resueltas punto a punto para hallar $\Delta H_{\text {soln }}^{\text {app }}$ a cada temperatura entre $15.0^{\circ} \mathrm{C}$ y $50.0^{\circ} \mathrm{C}$, valores de entalpía aparente que son presentados en la Tabla II.

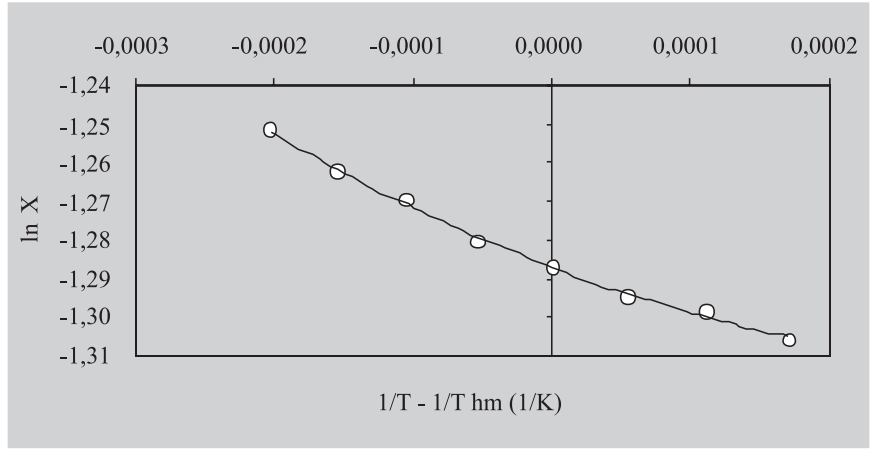

FIGURA 2 - Solubilidad del agua en el n-octanol en fracción molar en función de la temperatura considerando la temperatura armónica (303.15 K).

TABLA II - Entalpías aparentes $\left(\mathrm{J} \mathrm{mol}^{-1}\right)$ de solución del n-octanol en el agua y del agua en el n-octanol, obtenidas por el método parabólico de van’t Hoff y por el método de Grant et al. (1984) en función de la temperatura

\begin{tabular}{|c|c|c|c|c|}
\hline \multirow[t]{2}{*}{ Temp. $/{ }^{\circ} \mathrm{C}$} & \multicolumn{2}{|c|}{$n$-Octanol en agua } & \multicolumn{2}{|c|}{ Agua en $n$-octanol } \\
\hline & Van't Hoff & Grant et al. & Van't Hoff & Grant et al. \\
\hline 15.0 & 5294 & 5468 & 605.3 & 628.3 \\
\hline 20.0 & 6219 & 6294 & 783.9 & 790.7 \\
\hline 25.0 & 7113 & 7119 & 956.5 & 953.1 \\
\hline 30.0 & 7978 & 7945 & 1123.5 & 1115.6 \\
\hline 35.0 & 8815 & 8770 & 1285.0 & 1278.0 \\
\hline 40.0 & 9625 & 9596 & 1441.4 & 1440.5 \\
\hline 45.0 & 10410 & 10421 & 1592.8 & 1602.9 \\
\hline 50.0 & 11170 & 11247 & 1739.6 & 1765.3 \\
\hline
\end{tabular}


Los datos de la Tabla I también se procesaron de acuerdo a la Ec. 5 (Método de Grant et al:: Solubilidad en función de la temperatura absoluta) encontrando también coeficientes de determinación superiores a 0.997 usando este modelo (Anexo 2). Utilizando los coeficientes $a$ y $b$ se hallaron los valores de $\Delta H^{\text {app }}{ }_{\text {soln }}$ mediante la Ec. 6; estos valores también se presentan en la Tabla II. Puesto que en los dos casos se trata de la solubilidad de un soluto líquido en un solvente líquido para dar una solución líquida, la entalpía de solución corresponde básicamente a la entalpía de mezcla según el modelo clásico de solubilidad [solución = fusión + mezcla, en el caso de solutos sólidos (Martínez y Gómez, 2001)], siendo entonces procesos endotérmicos en los dos sistemas.

Comparando los valores $\Delta H^{\text {app }}{ }_{\text {soln }}$ obtenidos por los dos tratamientos realizados se encuentra que son prácticamente equivalentes, sin embargo debido al mayor uso en la literatura se decide continuar trabajando con los valores obtenidos por el primer método, esto es, regresión parabólica de $\ln X_{2}$ en función de $T^{-1}$, derivación y cálculo de las entalpías aparentes de solución.

En la Tabla III se presentan los valores para el cambio de energía libre estándar aparente de solución $\left(\Delta G^{\text {app }}{ }_{\text {soln }}\right)$ en función de la temperatura, los cuales se calcularon por medio de la Ec. 12, de la misma forma, en esta misma tabla se presentan los valores para el cambio entrópico estándar aparente de solución $\left(\Delta S_{\text {appln }}^{\text {app }}\right)$ en función de la temperatura, para los dos sistemas, que fueron calculados mediante la Ec. 13 a partir de las entalpías y energías libres aparentes. Sin embargo para hacer una interpretación más correcta de las posibles implicaciones de estas funciones termodinámicas a nivel molecular, estas deben ser corregidas por efecto de la posible variación de la actividad termodinámica con la concentración en solución, en particular los cambios entálpicos y entrópicos, lo cual se realiza por medio de las Ecs. 7 y 14, respectivamente.

\section{Cálculo de las funciones termodinámicas de solución corregidas}

Las funciones termodinámicas de solución corregidas se obtienen multiplicando los valores de las Tablas II y III por el factor $\left(\partial \ln a_{2} / \partial \ln X_{2}\right)_{T, P}$ el cual es calculado utilizando la Ec. 11 y cuyos valores se presentan en la Tabla IV. Tradicionalmente en la literatura (Martin y Bustamante, 1989) se ha utilizado como valor del volumen molar del soluto en la solución el correspondiente al compuesto puro $\left(V_{2}\right)$, sin embargo debe recordarse que en realidad aquí este valor correspondería más correctamente a la propiedad molar parcial; además, la fracción volumétrica del soluto en la solución saturada (requerido en la Ec. 11) se ha calculado según la expresión:

$$
\phi_{2}=\frac{V_{2} X_{2}}{V_{1}\left(1-X_{2}\right)+V_{2} X_{2}}
$$

Donde el término $V_{1}$ es el volumen molar del solvente. Como se indicó anteriormente, una aproximación más correcta aunque no exacta, es el uso de la Ec. 9 para hallar el valor $D_{2}$ que corresponde al volumen específico aparente (VEA). Estos dos valores se calcularon utilizando los datos de solubilidad y de densidad de las soluciones saturadas presentados en la Tabla I y además los datos de densidad del agua y del $n$-octanol puros tomados de la literatura (Budavari et al., 2001; Lide, 2003; Garzón, 2004). Los valores de $V E A$ y $\phi_{2}$ para los solutos en función de la temperatura se encuentran también en la Tabla IV.

En la Tabla V se presentan los valores para el cambio de energía libre estándar corregido de solución $\left(\Delta G_{\text {soln }}\right)$ en función de la temperatura, los cuales se calcularon multiplicando $\Delta G^{\text {app }}$ soln de la Tabla III por el factor $\left(\partial \ln a_{2} / \partial \ln \right.$ $\left.X_{2}\right)_{T, P}$ de la Tabla IV. Se puede observar en la Tabla V que en los dos sistemas, en todos los casos estudiados, esta propiedad es positiva lo cual es debido al estado de

TABLA III - Energías libres aparentes $\left(\mathrm{J} \mathrm{mol}^{-1}\right)$ y entropías aparentes $\left(\mathrm{J} \mathrm{mol}^{-1} \mathrm{~K}^{-1}\right)$ de solución del n-octanol en el agua y del agua en el n-octanol en función de la temperatura

\begin{tabular}{|c|c|c|c|c|}
\hline \multirow[t]{2}{*}{ Temp. $/{ }^{\circ} \mathrm{C}$} & \multicolumn{2}{|c|}{$n$-Octanol en agua } & \multicolumn{2}{|c|}{ Agua en $n$-octanol } \\
\hline & $\Delta G_{\text {soln }}^{\text {app }}$ & $\Delta S_{\text {soln }}^{\text {app }}$ & $\Delta G_{\text {soln }}^{\text {app }}$ & $\Delta S_{\text {soln }}^{\text {app }}$ \\
\hline 15.0 & 23541 & -63.33 & 3127.9 & -8.75 \\
\hline 20.0 & 23861 & -60.18 & 3164.2 & -8.12 \\
\hline 25.0 & 24139 & -57.10 & 3209.1 & -7.56 \\
\hline 30.0 & 24418 & -54.23 & 3244.6 & -7.00 \\
\hline 35.0 & 24700 & -51.55 & 3279.7 & -6.47 \\
\hline 40.0 & 24945 & -48.92 & 3304.9 & -5.95 \\
\hline 45.0 & 25159 & -46.36 & 3338.9 & -5.49 \\
\hline 50.0 & 25413 & -44.08 & 3363.1 & -5.02 \\
\hline
\end{tabular}


referencia utilizado, esto es, la solución ideal de fracción molar unitaria. Estos resultados pueden interpretarse en términos de la preferencia de los solutos por permanecer en su propia fase en lugar de pasar a la solución. Sin embargo, una consideración especial tendría que hacerse al calcular los valores $\Delta G^{\text {app }}{ }_{\text {soln }}$ en la escala de molaridad para la solubilidad del agua en el n-octanol (superior a 2.2 $\mathrm{mol} \mathrm{L}^{-1}$ ), ya que estos valores son negativos indicando la preferencia del agua por pasar a la solución en lugar de permanecer en su propia fase, además debe recordarse que en este segundo caso el estado de referencia es la solución ideal de concentración $1 \mathrm{~mol} \mathrm{~L}^{-1}$, que para el agua no es difícil de lograr debido a su masa molar relativamente pequeña (18.02 $\left.\mathrm{g} \mathrm{mol}^{-1}\right)$. El efecto de las escalas de concentración sobre los valores de las funciones termodinámicas de solución y de transferencia ha sido descrito en detalle previamente (Ben-Naim, 1978, 1979; Tanford, 1979; Martínez, 2001).

En los dos sistemas el proceso de solución es endotérmico y dependiente de la temperatura, requiriéndose más energía para disolver el $n$-octanol en el agua que para disolver el agua en el $n$-octanol, lo cual en principio podría interpretarse en términos de la mayor cantidad de energía requerida para crear una cavidad en el agua, del tamaño de la molécula de $n$-octanol, que la requerida para crear una cavidad en el $n$-octanol, del tamaño de la molécula de agua, recordando la mayor asociación existente en el agua respecto al $n$-octanol y además el tamaño muy superior de las moléculas de n-octanol respecto a las del agua.

De otro lado, se tiene que la entropía de solución es negativa en los dos sistemas estudiados y es también dependiente de la temperatura, encontrándose un mayor nivel de organización al disolver el $n$-octanol en el agua que en el caso contrario, debe recordarse que el proceso de solución como mezcla que es, en el caso ideal implica necesariamente un aumento en la entropía, esto es un aumento en el desorden a nivel molecular, sin embargo el hecho de encontrar entropías negativas da un indicativo de fenómenos de asociación muy marcados en las soluciones saturadas.

TABLA IV - Volumen específico aparente $\left(\mathrm{cm}^{3} \mathrm{~g}\right)$, fracción volumétrica y factor $\left(\partial \ln a_{2} / \partial \ln X_{2}\right)_{T, P}$ del $n$-octanol en el agua y del agua en el n-octanol en función de la temperatura

\begin{tabular}{lcccccc}
\hline Temp. $/{ }^{\circ} \mathbf{C}$ & \multicolumn{3}{c}{$\boldsymbol{n}$-Octanol en agua } & \multicolumn{3}{c}{ Agua en $\boldsymbol{n - o c t a n o l}$} \\
& $\boldsymbol{V} \boldsymbol{E} \boldsymbol{A}_{\mathrm{ROH}}$ & $\boldsymbol{\phi}_{\mathrm{ROH}}\left(\partial \ln \boldsymbol{a}_{\mathrm{ROH}} / \partial \ln \boldsymbol{X}_{\mathrm{ROH}}\right)$ & $\boldsymbol{V} \boldsymbol{E} \boldsymbol{A}_{\mathrm{W}}$ & $\boldsymbol{\phi}_{\mathrm{W}}$ & $\left(\partial \ln \boldsymbol{a}_{\mathrm{w}} / \partial \ln \boldsymbol{X}_{\mathrm{W}}\right)$ \\
\hline 15.0 & 1.1780 & 0.0004661 & 0.9908 & 0.9673 & 0.03900 & 0.8603 \\
20.0 & 1.1729 & 0.0004808 & 0.9906 & 0.9785 & 0.03966 & 0.8584 \\
25.0 & 1.1657 & 0.0005029 & 0.9902 & 0.9863 & 0.04000 & 0.8574 \\
30.0 & 1.1597 & 0.0005249 & 0.9898 & 0.9925 & 0.04047 & 0.8561 \\
35.0 & 1.1759 & 0.0005571 & 0.9893 & 1.0056 & 0.04121 & 0.8539 \\
40.0 & 1.1885 & 0.0005966 & 0.9886 & 1.0135 & 0.04195 & 0.8519 \\
45.0 & 1.1603 & 0.0006234 & 0.9881 & 1.0230 & 0.04256 & 0.8501 \\
50.0 & 1.1912 & 0.0006731 & 0.9873 & 1.0333 & 0.04340 & 0.8478 \\
\hline
\end{tabular}

TABLA V - Energías libres corregidas $\left(\mathrm{J} \mathrm{mol}^{-1}\right)$, entalpías corregidas $\left(\mathrm{J} \mathrm{mol}^{-1}\right)$ y entropías corregidas $\left(\mathrm{J}\right.$ mol $\left.{ }^{-1} \mathrm{~K}^{-1}\right)$ de solución del $n$-octanol en el agua y del agua en el $n$-octanol en función de la temperatura

\begin{tabular}{lcccccc}
\hline Temp. $~^{\circ} \mathbf{C}$ & \multicolumn{3}{c}{$\boldsymbol{n}$-Octanol en agua } & \multicolumn{3}{c}{ Agua en $\boldsymbol{n}$-octanol } \\
& $\boldsymbol{\Delta} \boldsymbol{G}_{\text {soln }}$ & $\boldsymbol{\Delta} \boldsymbol{H}_{\text {soln }}$ & $\boldsymbol{\Delta} \boldsymbol{S}_{\text {soln }}$ & $\boldsymbol{\Delta} \boldsymbol{G}_{\text {soln }}$ & $\boldsymbol{\Delta} \boldsymbol{H}_{\text {soln }}$ & $\boldsymbol{\Delta} \boldsymbol{S}_{\text {soln }}$ \\
\hline 15.0 & 23326 & 5245 & -62.75 & 2691.0 & 520.7 & -7.53 \\
20.0 & 23636 & 6161 & -59.61 & 2716.0 & 672.9 & -6.97 \\
25.0 & 23902 & 7044 & -56.54 & 2751.4 & 820.1 & -6.48 \\
30.0 & 24170 & 7897 & -53.68 & 2777.7 & 961.8 & -5.99 \\
35.0 & 24435 & 8720 & -51.00 & 2800.4 & 1097.2 & -5.53 \\
40.0 & 24660 & 9515 & -48.36 & 2815.4 & 1227.9 & -5.07 \\
45.0 & 24860 & 10286 & -45.81 & 2838.6 & 1354.1 & -4.67 \\
50.0 & 25089 & 11028 & -43.51 & 2851.3 & 1474.9 & -4.26 \\
\hline
\end{tabular}


En el caso del agua saturada de $n$-octanol, con un cambio entrópico de $-56.5 \mathrm{~J} \mathrm{~mol}^{-1} \mathrm{~K}^{-1}$ implica una gran restricción de libertad molecular la cual puede atribuirse a la hidratación hidrofóbica, esto es, la autoasociación de moléculas de agua alrededor de las cadenas octílicas del soluto para generar agua voluminosa, lo cual disminuye la libertad de movilidad del agua en la vecindad de las moléculas del soluto. De otro lado, la ligera reducción entrópica en el n-octanol saturado de agua que compensa plenamente el aumento entrópico debido al proceso de mezcla, implica también algún tipo de ordenamiento molecular, el cual ha sido explicado como se indicó anteriormente en términos de la estructura microheterogénea de este sistema, el cual en la saturación presenta básicamente la asociación por enlace de hidrógeno de dos moléculas de agua, y cada una de estas a su vez unida por enlace de hidrógeno a tres moléculas de noctanol, con lo cual resulta en promedio una molécula de agua por tres moléculas de $n$-octanol, llevando en términos molares a una composición $X_{\mathrm{w}} 0.25$ y $X_{\mathrm{ROH}} 0.75$, valor próximo al presentado en la Tabla I, e incluso más cercano al presentado por otros autores citados por Sangster (1997). Como se ha indicado ampliamente en la literatura, es precisamente debido a este carácter microheterogéneo del noctanol saturado de agua que resulta un excelente sistema de reparto para uso en estudios QSAR.

Con el ánimo de evaluar los resultados entálpicos obtenidos en el tratamiento de los datos de equilibrio del sistema $n$-octanol saturado de agua, se tiene que a $25.0^{\circ} \mathrm{C}$ la entalpía de solución corregida es $0.82 \mathrm{~kJ} \mathrm{~mol}^{-1} \mathrm{y}$ sin corregir es $0.96 \mathrm{~kJ} \mathrm{~mol}^{-1}$, mientras que el valor correspondiente obtenido por calorimetría isoperibólica es $3.44 \pm 0.07 \mathrm{~kJ} \mathrm{~mol}^{-1}$ (Berti et al., 1986; lo cual lleva a un valor $\Delta S_{\text {soln }}$ de $0.77 \mathrm{~J}$ $\mathrm{mol}^{-1} \mathrm{~K}^{-1}$, usando los datos de $\Delta G_{\text {soln }}$ sin corregir de la Tabla III a $25.0^{\circ} \mathrm{C}: 3.21 \mathrm{~kJ} \mathrm{~mol}^{-1}$ ). De acuerdo a esto, usando datos calorimétricos se tendría un ligero aumento del desorden en el $n$-octanol saturado de agua, infortunadamente en términos termodinámicos clásicos no es mucho lo que se puede inferir respecto a la estructura y organización molecular en este sistema, el cual ha sido abordado por DeBolt y Kollman(1995) desde otro tipo de tratamiento mediante consideraciones teóricas. A diferencia del caso anterior, no existen datos calorimétricos reportados sobre la entalpía de solución del noctanol en el agua.

Como una ampliación de la información termodinámica basada en el método de van't Hoff sobre estos dos sistemas, se procesaron adicionalmente otros datos presentados por Sangster (1997), en particular aquellos que recomienda personalmente este autor.

Los valores de solubilidad del agua en el $n$-octanol presentados por Hefter (1984) citados por Sangster (1997), junto con las correspondientes funciones termodinámicas aparentes de solución se presentan en la Tabla VI, mientras que la misma información basada en los datos de Sorensen y Artl (1979) citados por Sangster (1997) se presenta en la Tabla VII. En la Figura 3, con fines comparativos se presentan gráficamente estos valores

TABLA VI - Solubilidad del agua en el n-octanol y funciones termodinámicas aparentes de solución en función de la temperatura según los datos de Hefter (1984) citados por Sangster (1997)

\begin{tabular}{lcccc}
\hline Temp. $/{ }^{\circ} \mathbf{C}$ & $\boldsymbol{X}_{\mathrm{W}}$ & $\Delta \boldsymbol{G}_{\text {soln }}^{\text {app }}(\mathbf{a})$ & $\Delta \boldsymbol{H}_{\text {soln }}^{\text {app }}(\mathbf{a})$ & $\Delta \boldsymbol{S}_{\text {soln }}^{\text {app }}(\mathbf{a})$ \\
\hline 20.0 & 0.257 & 3086 & 2134 & -3.24 \\
25.0 & 0.261 & 3051 & 2916 & -0.45 \\
30.0 & 0.266 & 3007 & 3671 & 2.19 \\
40.0 & 0.283 & 2867 & 5110 & 7.16 \\
60.0 & 0.327 & 2539 & 7728 & 15.6 \\
\hline
\end{tabular}

(a). Unidades: $\Delta G_{\text {soln }}^{\text {app }} \Delta H_{\text {soln }}^{\text {app }} \mathrm{J} \mathrm{mol}^{-1} ; \Delta S_{\text {soln }}^{\text {app }}: \mathrm{J} \mathrm{mol}^{-1} \mathrm{~K}^{-1}$

TABLA VII - Solubilidad del agua en el n-octanol y funciones termodinámicas aparentes de solución en función de la temperatura según los datos de Sorensen y Artl (1979) citados por Sangster (1997)

\begin{tabular}{lcccc}
\hline Temp. $/{ }^{\circ} \mathrm{C}$ & $X_{\mathrm{w}}$ & $\Delta G_{\text {soln }}^{\text {app }}(\mathrm{a})$ & $\Delta H^{\text {app }}{ }_{\text {soln }}(\mathrm{a})$ & $\Delta S_{\text {soln }}^{\text {app }}(\mathrm{a})$ \\
\hline 20.0 & 0.194 & 3724 & 10247 & 22.3 \\
25.0 & 0.207 & 3577 & 9713 & 20.6 \\
40.0 & 0.247 & 3176 & 8214 & 16.1 \\
60.0 & 0.292 & 2796 & 6424 & 10.9 \\
\hline
\end{tabular}

(a). Unidades: $\Delta G_{\text {soln }}^{\text {app }} \Delta H_{\text {soln }}^{\text {app }} \mathrm{J} \mathrm{mol}^{-1} ; \Delta S_{\text {soln }}^{\text {app }}: \mathrm{J} \mathrm{mol}^{-1} \mathrm{~K}^{-1}$ 


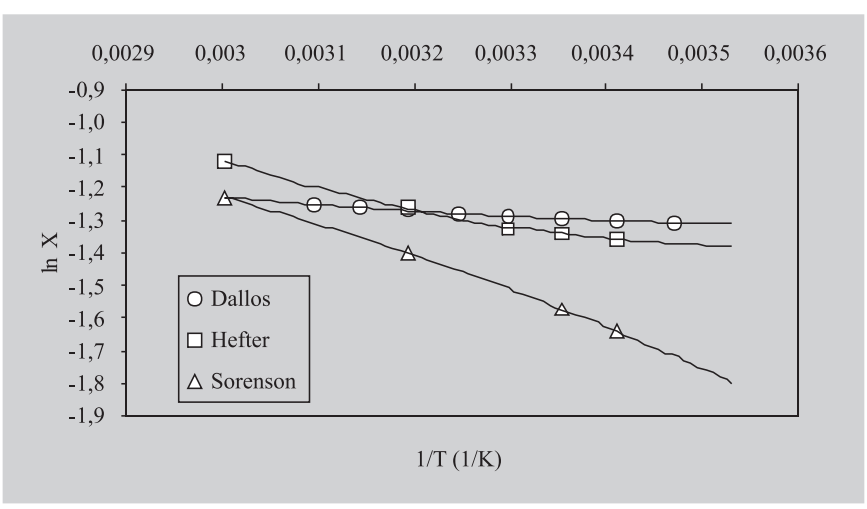

FIGURA 3 - Solubilidad del agua en el $n$-octanol en función de la temperatura según los datos de Dallos y Liszi (1995), de Hefter (1984) citados por Sangster (1997), y de Sorensen y Artl (1979) citados por Sangster (1997).

de solubilidad junto a los de Dallos y Liszi (1995) y además, las ecuaciones de regresión se presentan en el Anexo 3.

Comparando los resultados de las Tablas III (Dallos y Liszi, 1995), VI (Hefter, 1984) y VII (Sorensen y Artl, 1979: valores recomendados por Sangster, 1997), se observa que los valores de $\Delta G^{\text {app }}{ }_{\text {soln }}$ aumentan al aumentar la temperatura según los datos de Dallos y Liszi mientras que el comportamiento es contrario en las otras dos referencias; de otro lado los valores de $\Delta H^{\text {app }}{ }_{\text {soln }}$ aumentan al aumentar la temperatura según los datos de Dallos y Liszi y de Hefter mientras que el comportamiento es contrario con los datos de Sorensen y Artl, finalmente, los valores de $\Delta S^{\text {app }}$ soln aumentan al aumentar la temperatura según los datos de Dallos y Liszi y de Hefter mientras que nuevamente el comportamiento es contrario con los datos de Sorensen y Artl.

Respecto a la concordancia de valores termodinámicos a $25.0 \mathrm{C}$ se tiene que el cambio de energía libre aparente de solución es muy similar, sin embargo los cambios entálpico aparente y entrópico aparente de solución son muy variables, esto es para la entalpía: 0.96 $\mathrm{kJ} \mathrm{mol}^{-1}, 2.92 \mathrm{~kJ} \mathrm{~mol}^{-1}$ y $9.71 \mathrm{~kJ} \mathrm{~mol}^{-1}$, y para la entropía: $-7.56 \mathrm{~J} \mathrm{~mol}^{-1} \mathrm{~K}^{-1},-0.45 \mathrm{~J} \mathrm{~mol}^{-1} \mathrm{~K}^{-1}$ y $20.6 \mathrm{~J} \mathrm{~mol}^{-1} \mathrm{~K}^{-1}$, para los datos de Dallos y Liszi, Hefter, y Sorensen y Artl, respectivamente. Lo anterior refleja claramente el efecto de la variación encontrada en los datos de equilibrio en función de la temperatura obtenidos por diferentes experimentadores, sin embargo puede observarse que los valores de entalpía y entropía más concordantes con los obtenidos por calorimetría son los obtenidos con los datos de solubilidad presentados por Hefter $\left(2.92 \mathrm{~kJ} \mathrm{~mol}^{-1}\right.$ frente a $3.44 \mathrm{~kJ} \mathrm{~mol}^{-1} \mathrm{y}-0.45 \mathrm{~J} \mathrm{~mol}^{-1} \mathrm{~K}^{-1}$ frente a $0.77 \mathrm{~J} \mathrm{~mol}^{-1}$ $\mathrm{K}^{-1}$ ). Adicionalmente se procesaron los datos presentados por otros investigadores, en particular los de Stephenson et al. (1984) citados por Sangster (1997), los cuáles conducen a valores de $\Delta G^{\text {app }}{ }_{\text {soln }} 3440 \mathrm{~J} \mathrm{~mol}^{-1}, \mathrm{D} H^{\text {app }}$ soln $2492 \mathrm{~J} \mathrm{~mol}^{-1}$ y $\Delta S_{\text {soln }}^{\text {app }}-3.12 \mathrm{~J} \mathrm{~mol}^{-1} \mathrm{~K}^{-1}$ a $25.0^{\circ} \mathrm{C}$, y los de von Erichsen (1952), citados también por Sangster (1997), los cuáles a su vez, conducen a valores de $\Delta G^{\text {app }}$ $3347 \mathrm{~J} \mathrm{~mol}^{-1}, \Delta H_{\text {soln }}^{\text {app }} 6143 \mathrm{~J} \mathrm{~mol}^{-1}$ y $\Delta S_{\text {soln }}^{\text {app }} 9.22 \mathrm{~J} \mathrm{~mol}^{-1}$ $\mathrm{K}^{-1}$ a $30.0^{\circ} \mathrm{C}$. Las ecuaciones correspondientes se presentan también en el Anexo 3. De estos últimos dos investigadores, el dato de entalpía más concordante con el obtenido por calorimetría es el de Stephenson et al.

Como se indicó anteriormente, no se dispone de datos calorimétricos para el proceso de solución del noctanol en el agua, sin embargo con fines de comparación respecto a los valores de Dallos y Liszi (1995), en las Tablas VIII y IX se presentan los valores de solubilidad de n-octanol en agua reportados por Sorensen y Artl (1979) y por Lebedinskaya et al. (1984) citados también por Sangster (1997), respectivamente, junto con los correspondientes valores de las funciones termodinámicas de solución; de la misma forma, estos resultados se presentan gráficamente en la Figura 4, mientras que las ecuaciones de regresión se presentan en el Anexo 3. Puede observarse que los datos más concordantes con los de Dallos y Liszi son los presentados por Lebedinskaya et al., aún cuando los datos de Sorensen y Artl sean los recomendados por Sangster (1997) en su texto clásico de reparto.

Recientemente Perlovich et al. (2004), introdujeron en la termodinámica de soluciones farmacéuticas la idea de analizar el proceso de solución de solutos sólidos en

TABLA VIII - Solubilidad del $n$-octanol en el agua y funciones termodinámicas aparentes de solución en función de la temperatura según los datos de Sorensen y Artl (1979) citados por Sangster (1997)

\begin{tabular}{lcccc}
\hline Temp. $/{ }^{\circ} \mathrm{C}$ & $X_{\mathrm{ROH}}\left(10^{5}\right)$ & $\Delta G_{\text {soln }}^{\text {app }}(\mathrm{a})$ & $\Delta H^{\text {app }}{ }_{\text {soln }}(\mathrm{a})$ & $\Delta S_{\text {soln }}^{\text {app }}(\mathrm{a})$ \\
\hline 20.0 & 6.25 & 23593 & 25906 & 8.46 \\
25.0 & 7.03 & 23704 & 25906 & 8.06 \\
40.0 & 14.4 & 23030 & 25906 & 10.5 \\
60.0 & 21.1 & 23443 & 25906 & 9.02 \\
\hline
\end{tabular}

(a). Unidades: $\Delta G^{\text {app }}{ }_{\text {soln }}$ y $\Delta H^{\text {app }}{ }_{\text {soln }} . \mathrm{J} \mathrm{mol}^{-1} ; \Delta S_{\text {soln }}^{\text {app }}: \mathrm{J} \mathrm{mol}^{-1} \mathrm{~K}^{-1}$ 
TABLA IX - Solubilidad del n-octanol en el agua y funciones termodinámicas aparentes de solución en función de la temperatura según los datos de Lebedinskaya et al. (1984) citados por Sangster (1997)

\begin{tabular}{lcccc}
\hline Temp. $/{ }^{\circ} \mathrm{C}$ & $X_{\mathrm{ROH}}\left(10^{5}\right)$ & $\Delta G_{\text {soln }}^{\text {app }}(\mathrm{a})$ & $\Delta H_{\text {soln }}^{\text {app }}(\mathrm{a})$ & $\Delta S_{\text {soln }}^{\text {app }}(\mathrm{a})$ \\
\hline 20.0 & 5.4 & 23950 & 11848 & -41.3 \\
40.0 & 7.2 & 24835 & 11848 & -41.5 \\
60.0 & 9.0 & 25803 & 11848 & -41.9 \\
80.0 & 12.6 & 26364 & 11848 & -41.1 \\
\hline
\end{tabular}

(a). Unidades: $\Delta G_{\text {soln }}^{\text {app }} \Delta H_{\text {soln }}^{\text {app }} \mathrm{J} \mathrm{mol}^{-1} ; \Delta S_{\text {soln }}^{\text {app }}: \mathrm{J} \mathrm{mol}^{-1} \mathrm{~K}^{-1}$

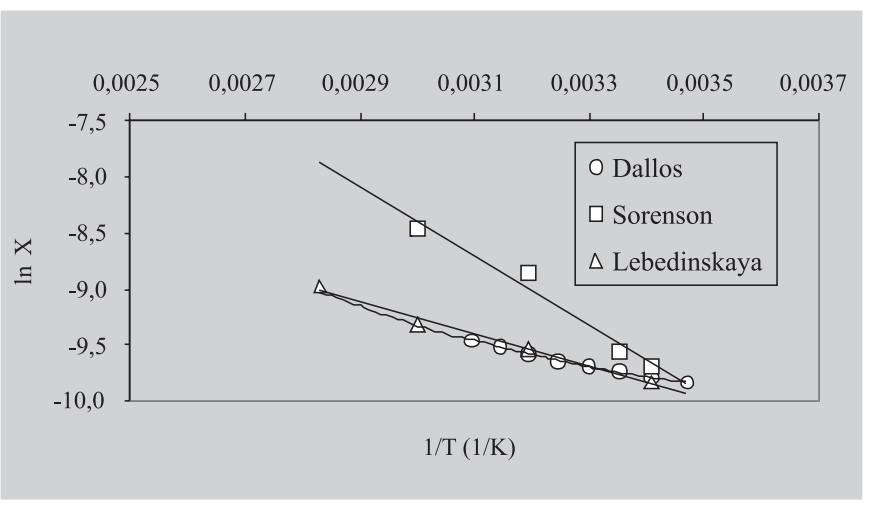

FIGURA 4 - Solubilidad del $n$-octanol en el agua en función de la temperatura según los datos de Dallos y Liszi (1997), de Lebedinskaya et al. (1984) citados por Sangster (1997), y de Sorensen y Artl (1979) citados por Sangster (1997).

términos de los subprocesos hipotéticos: sublimación y solvatación, en adición al más clásico, de fusión y mezcla. Puesto que en los dos sistemas bajo estudio, como se indicó previamente, los solutos son líquidos y por lo tanto el proceso de solución es equivalente al de mezcla (no hay fusión del soluto), entonces en nuestro caso el tratamiento de Perlovich et al., puede realizarse con datos de vaporización para obtener las funciones termodinámicas: energía libre, entalpía y entropía de solvatación, mediante las siguientes expresiones:

$$
\begin{gathered}
\Delta G_{\text {solv }}=\Delta G_{\text {soln }}-\Delta G_{\text {vap }} \\
\Delta H_{\text {solv }}=\Delta H_{\text {soln }}-\Delta H_{\text {vap }} \\
\Delta S_{\text {solv }}=\Delta S_{\text {soln }}-\Delta S_{\text {vap }}
\end{gathered}
$$

Las funciones termodinámicas de vaporización para el agua y el n-octanol se calcularon a partir de valores de presión de vapor en función de la temperatura reportados en la literatura (Liley et al., 1992), los cuales fueron procesados por el método de Clausius-Clapeyron (ecuación de van’t Hoff para el equilibrio líquido-vapor), usando una expresión análoga a la Ec. 1 para hallar la entalpía aparente (Anexo 4), mientras que la energía libre fue calculada mediante una expresión análoga a la Ec. 12, utilizando como estado estándar la presión de $1 \mathrm{~atm}$. La entropía correspondiente se calculó mediante una expresión análoga a la Ec. 13. Del tratamiento anterior se obtuvieron los siguientes valores a $25.0^{\circ} \mathrm{C}$ para el agua: $\Delta G_{\text {vap }} 8590 \mathrm{~J} \mathrm{~mol}^{-1}, \Delta H_{\text {vap }} 43257 \mathrm{~J} \mathrm{~mol}^{-1}$ y $\Delta S_{\text {vap }} 116.27 \mathrm{~J}$ $\mathrm{mol}^{-1} \mathrm{~K}^{-1}$, y en el caso del n-octanol: $\Delta G_{\text {vap }} 22751 \mathrm{~J} \mathrm{~mol}^{-1}$, $\Delta H_{\text {vap }} 74149 \mathrm{~J} \mathrm{~mol}^{-1}$ y $\Delta S_{\text {vap }} 172.39 \mathrm{~J} \mathrm{~mol}^{-1} \mathrm{~K}^{-1}$.

Respecto a los valores termodinámicos de solución, en el caso de la solubilidad del $n$-octanol en el agua, se utilizaron los valores corregidos obtenidos mediante el tratamiento de los datos de Dallos y Liszi (1995), y en el caso de la solubilidad del agua en el n-octanol, se consideró la energía libre aparente a $25.0^{\circ} \mathrm{C}$ según Dallos y Liszi (Tabla III), mientras que la entalpía de solución utilizada fue la obtenida mediante calorimetría por Berti et al. (1986), y por lo tanto la entropía utilizada se obtuvo usando estos dos valores.

En la Tabla X se presentan los valores termodinámicos de solvatación para los sistemas en estudio, los cuales se calcularon por medio de las Ecs. 18 a 20, usando los valores indicados anteriormente. Adicionalmente Perlovich et al. (2004) han utilizado las Ecs. 21 y 22 para evaluar la contribución porcentual de los términos entálpico $(H)$ y entrópico $(T S)$ a la energía libre de solvatación, lo cual en principio también puede aportar información sobre los fenómenos presentados a nivel molecular cuando las moléculas de soluto son transferidas desde el estado de vapor hasta la solución, por lo cual en la esta misma tabla se presentan los valores de $\% z_{H}$ y $\% z_{T S}$ para los sistemas estudiados.

$$
\begin{aligned}
& \% \zeta_{H}=100 \frac{\left|\Delta H_{\text {solv }}\right|}{\left|\Delta H_{\text {solv }}\right|+\left|T \Delta S_{\text {solv }}\right|} \\
& \% \zeta_{T S}=100 \frac{\left|T \Delta S_{\text {solv }}\right|}{\left|\Delta H_{\text {solv }}\right|+\left|T \Delta S_{\text {solv }}\right|}
\end{aligned}
$$


TABLA X - Funciones termodinámicas de solvatación del $n$-octanol en el agua y del agua en el $n$-octanol a $25.0{ }^{\circ} \mathrm{C}$

\begin{tabular}{lcccccc}
\hline Sistema & $\Delta G_{\text {solv }}(\mathrm{a})$ & $\Delta H_{\text {solv }}(\mathrm{a})$ & $\Delta S_{\text {solv }}(\mathrm{a})$ & $T \Delta S_{\text {solv }}$ (a) & $\% \xi_{H}$ & $\% \xi_{T S}$ \\
\hline $\mathrm{W}_{(\mathrm{ROH})}$ & 1151 & -67105 & -228.9 & -68255 & 49.58 & 50.42 \\
$\mathrm{ROH}_{(\mathrm{W})}$ & -5839 & -39817 & -115.5 & -34437 & 53.62 & 46.38 \\
\hline
\end{tabular}

(a). Unidades: $\Delta G_{\text {solv }}, \Delta H_{\text {solv }}$ y $T \Delta S_{\text {solv }}$. $\mathrm{mol}^{-1} ; \Delta S_{\text {solv }}: \mathrm{J} \mathrm{mol}^{-1} \mathrm{~K}^{-1}$

De los datos de $\Delta G_{\text {solv }}$ para el caso de la hidratación del $n$-octanol $\left(\mathrm{W}_{(\mathrm{ROH})}\right)$, debido al valor positivo de esta propiedad termodinámica se tiene que el $n$-octanol en principio presentaría mayor afinidad por la fase de vapor que por la solución acuosa, mientras que en el caso de la solvatación del agua en el n-octanol $\left(\mathrm{ROH}_{(\mathrm{w})}\right)$ la situación es contraria, esto es, el agua prefiere la solución en el noctanol que permanecer en la fase de vapor. Como es de esperarse debido al paso de los solutos del estado de vapor a la fase condensada de las soluciones, en los dos casos, las funciones $\Delta H_{\text {solv }}$ y $\Delta S_{\text {solv }}$ presentan valores negativos debido al establecimiento de uniones entre las moléculas del soluto y las moléculas del solvente (interacciones soluto-solvente) y en el caso de la solvatación del agua en el n-octanol, como se indicó anteriormente, también debido a la formación de la unión agua-agua (interacciones solutosoluto), lo que conduce a la liberación de energía y a la reducción de la libertad de movilidad molecular.

En cuanto a la contribución energética y organizacional al proceso de solvatación de los dos solutos considerados, se tiene que para la hidratación del $n$-octanol la participación es prácticamente a partes iguales, mientras que en la solvatación del agua, se presenta un ligero predominio energético, lo cual en el primer caso mencionado $\left(\mathrm{W}_{(\mathrm{ROH})}\right)$, puede deberse en gran medida a la autoasociación del agua en torno a las cadenas octílicas (hidratación hidrofóbica) que igualaría en contribución al aporte energético, mientras que en el segundo caso $\left(\mathrm{ROH}_{(\mathrm{W})}\right)$, debido a la formación de un alto número de enlaces de hidrógeno durante la formación de la estructura microheterogénea orgánica discutida previamente, el componente energético primaría sobre el organizacional a nivel molecular y más si se considera que la cavidad requerida en este caso es mucho más pequeña que la requerida en el caso de la hidratación del $n$-octanol.

De todo lo expuesto anteriormente se puede concluir que la información existente sobre el equilibrio líquido-líquido entre el $n$-octanol y el agua en función de la temperatura es muy amplia, sin embargo en la mayoría de los casos no hay concordancia en los trabajos de los diferentes investigadores, lo cual como se ha observado lleva a estimaciones de las funciones termodinámicas casi completamente diferentes. A pesar de lo inmediatamente mencionado y gracias a la existencia de otros tratamientos extratermodinámicos y teóricos, en términos generales puede concluirse que las funciones termodinámicas calculadas a partir de los datos de Dallos y Liszi en combinación con otros datos procedentes de la literatura permiten corroborar lo expuesto previamente en la literatura sobre la estructuración del agua en torno a las cadenas alquílicas de solutos "hidrófobos" y además, sobre la estructura microheterogénea presente en el $n$-octanol saturado de agua. Sería muy interesante disponer de datos calorimétricos sobre la entalpía de disolución del n-octanol en el agua, lo cual permitiría corroborar la validez del método de van't Hoff para la estimación de funciones termodinámicas de solución en medios acuosos.

\section{ABSTRACT}

\section{Thermodynamic aspects of partial miscibility between $n$-octanol and water}

In the present work the thermodynamic analysis for those data presented by Dallos and Liszi on mole fraction n-octanol-water liquid-liquid equilibria was made. The values were analyzed using the van't Hoff method ( $\ln S$ as a function of $T^{-1}$ ) and those presented by Grant et al. (In $S$ as a function of $T^{-1}$ and $\left.\ln T\right)$. A non-lineal behavior was found in all cases studied by the van't Hoff method, which lead us to apply a parabolic regression model derived in order to calculate the enthalpic changes. Endothermic processes were obtained for all temperatures studied. In both systems the free energy changes were positive, whereas the entropic changes were negative indicating some kind of organization in the saturated solutions. In the case of n-octanol-saturated water this result would be explained as the hydrophobic hydration around the aliphatic groups and on the other hand, in the case of water-saturated n-octanol, it could be due to organization of n-octanol molecules around water molecules by hydrogen bonds as it has been presented in literature. The thermodynamic values calculated were compared with those presented by other authors, which were obtained by 
calorimetry and also by means of equilibrium constants as a function of temperature.

UNITERMS: n-Octanol-water system, Partial miscibility, Solution thermodynamics

\section{BIBLIOGRAFÍA}

ÁVILA, C.M.; MARTÍNEZ, F. Thermodynamics of partitioning of benzocaine in some organic solvent/buffer and liposome systems. Chem. Pharm. Bull., Tokyo, v. 51, n. 3, p. 237-240, 2003.

BAENA, Y.; PINZÓN, J.; BARBOSA, H.; MARTÍNEZ, F. Estudio termodinámico de la transferencia de acetaminofén desde el agua hasta el octanol. Rev. Bras. Cienc. Farm., Sao Paulo, v. 40, n. 3, p. 413-420, 2004.

BEN-NAIM, A. Standard thermodynamics of transfer. Uses and misuses. J. Phys. Chem., Washington, v. 82, n. 7, p. 792-803, 1978.

BEN-NAIM, A. Reply to C. Tanford's comments concerning standard states in the thermodynamics of transfer. $J$. Phys. Chem., Washington, v. 83, n. 13, p. 1803, 1979.

BERTI, P.; CABANI, S.; CONTI, G.; MOLLICA, V. Thermodynamic study of organic compounds in octan-1ol. J. Chem. Soc., Faraday Trans. 1, London, v. 82, p. 2547-2556, 1986.

BUDAVARI, S.; O'NEIL, M.J.; SMITH, A.; HECKELMAN, P.E.; OBENCHAIN Jr., J.R.; GALLIPEAU, J.A.R.; D'ARECEA, M.A. The Merck Index: An encyclopedia of chemicals, drugs, and biologicals. 13. ed. Whitehouse Station: NJ Merck \& Co., Inc., 2001.p. 1210-1211, 1791.

BUSTAMANTE, P.; ROMERO, S.; PEÑA, A.; REILLO, A. Enthalpy-entropy compensation for the solubility of drugs in solvent mixtures: Paracetamol, acetanilide, and nalidixic acid in dioxane-water. J. Pharm. Sci., Washington, v. 87, n. 12, p. 1590-1596, 1998.

CHUMAN, H.; MORI, A.; TANAKA, H. Prediction of the 1-octanol/ $\mathrm{H}_{2} \mathrm{O}$ partition coefficient, $\log P$, by Ab Initio MO calculations: Hydrogen-bonding effect of organic solutes on $\log$ P. Anal. Sci., Tokyo, v. 18, p. 1015-1020, 2002.
DALLOS, A.; LISZI, J. (Liquid + liquid) equilibria of (octan1-ol + water) at temperatures from $288.15 \mathrm{~K}$ to $323.15 \mathrm{~K}$. J. Chem. Thermodynamics, Amsterdam, v. 27, n. 4, p. 447-448, 1995.

DEBOLT, S.E.; KOLLMAN, P.A. Investigation of structure, dynamics, and solvation in 1-octanol and its watersaturated solution: molecular dynamics and free-energy perturbation studies. J. Am. Chem. Soc., Washington, v. 117, p. 5316-5340, 1995.

FRANKS, N.P.; ABRAHAM, M.H.; LIEB, W.R. Molecular organization of liquid n-octanol: An X-ray diffraction analysis. J. Pharm. Sci., Washington, v. 82, n. 5, p. 466470, 1993.

GARZÓN, L.C.A. Estudio termodinámico de la solubilidad del ibuprofeno en algunos solventes orgánicos y acuosos. Bogotá D.C., 2004. 67p. [Trabajo de Grado Departamento de Farmacia-Universidad Nacional de Colombia].

GRANT, D.J.W.; MEHDIZADEH, M.; CHOW, A.H.L.; FAIRBROTHER, J.E. Nonlinear van't Hoff solubilitytemperature plots and their pharmaceutical interpretation. Int. J. Pharm., Amsterdam, v. 18, p. 25-38, 1984.

HANSCH, C.; LEO, A. Exploring QSAR: Fundamentals and applications in chemistry and biology. Washington: American Chemical Society, 1995. p. 97-124.

HEFTER, G.T. (1984). Solubility Data Ser., v. 15, p. 364. apud SANGSTER, J. Octanol-Water Partition Coefficients: Fundamentals and Physical Chemistry. Chichester: John Wiley \& Sons, 1997. p. 1-55.

HOLLENBECK, R.G. Determination of differential heat of solution in real solutions from variation in solubility with temperature. J. Pharm. Sci., Washington, v. 69, n. 10, p. 1241-1242, 1980.

KRISTL,A.; VESNAVER, G. Thermodynamic investigation of the effect of octanol-water mutual miscibility on the partitioning and solubility of some guanine derivatives. $J$. Chem. Soc. Faraday Trans., London, v. 91, n. 6, p. 995998, 1995.

KRUG, R.R.; HUNTER, W.G.; GRIEGER, R.A. Enthalpyentropy compensation. 2. Separation of the chemical from the statistical effects. J. Phys. Chem., Washington, v. 80, p. 2341-2351, 1976. 
LEBEDINSKAYA, N.A. ; KUSHNER, T.M.; IVANSKAYA, L.N. (1984). Khim. Prom-st, Moscú, v. 4, p. 206. apud SANGSTER, J. Octanol-water partition coefficients: fundamentals and physical chemistry. Chichester: John Wiley \& Sons, 1997. p. 1-55.

LEO, A.; HANSCH, C.; ELKINS, D. Partition coefficients and their uses. Chem. Rev., Washington, v. 71, n. 6, p. 525$616,1971$.

LIDE, D.R. CRC Handbook of chemistry and physics. 84. ed. Boca Raton: CRC Press LLC, 2003. p. 3-440.

LILEY, P.E.; REID, R.C.; BUCK, E. Datos físicos y químicos. En: PERRY, R.H.; GREEN, D.W.; MALONEY, J.O., ed. Perry: Manual del ingeniero químico. 6. ed. México: McGraw-Hill, 1992. Vol 1. Sección 3.

MANZO, R.H.; AHUMADA, A.A. Effects of solvent medium on solubility. V. Enthalpic and entropic contributions to the free energy changes of di-substituted benzene derivatives in ethanol:water and ethanol:cyclohexane mixtures J. Pharm. Sci., Washington, v. 79, n. 12, p. 1109-1115, 1990.

MARTIN, A.; BUSTAMANTE, P. El parámetro de solubilidad en las ciencias farmacéuticas. Anal. Real Acad. Farm., Madrid, v. 55, n. 2, p. 175-202, 1989.

MARTIN, A.; BUSTAMANTE, P.; CHUN, A.H.C. Physical pharmacy: physical chemical principles in the pharmaceutical sciences. 4. ed. Philadelphia: Lea \& Febiger, 1993. p. 87.

MARTÍNEZ, F.; TELLO, M.; GÓMEZ, A. Solventes orgánicos como sistemas de reparto en modelación QSAR. Rev. Col. Cienc. Quím. Farm., Bogotá, v. 29, p. $16-25,2000$.

MARTÍNEZ, F. Aspectos termodinámicos de la transferencia de sulfonamidas entre medios acuosos y sistemas orgánicos. Bogotá, D.C., 2001. 271p. Tesis de Doctorado -Departamento de Química-Universidad Nacional de Colombia.

MARTÍNEZ, F.; ROJAS, J.; PERILLA, J.; GÓMEZ, A. Efecto de la miscibilidad parcial entre octanol y agua sobre la solubilidad y el reparto de algunas sulfonamidas. Rev. Col. Quím., Bogotá, v. 30, n. 1, p. 59-75, 2001.
MARTÍNEZ, F.; GÓMEZ, A. Thermodynamic study of the solubility of some sulfonamides in octanol, water, and the mutually saturated solvents. J. Solution Chem., New York, v. 30, n. 10, p. 909-923, 2001.

MARTÍNEZ, F.; ROJAS, J.H.; GÓMEZ, A. Thermodynamic study of partitioning of sulfanilamide and sulfamethoxazole at two pH values. Rev. Col. Cienc. Quím. Farm., Bogotá, v. 30, p. 35-42, 2001.

MARTÍNEZ, F.; GÓMEZ,A. Thermodynamics of partitioning of some sulfonamides in 1-octanol/buffer and liposome systems. J. Phys. Org. Chem., New York, v. 15, n. 12, p. 874-880, 2002.

PÉREZ, D.C.; GUEVARA, C.C.; CÁRDENAS, C.A.; PINZÓN, J.A.; BARBOSA, H.J.; MARTÍNEZ, F. Solubilidad y volúmenes de desplazamiento del acetaminofén en mezclas binarias formadas por agua, etanol y propilenoglicol a $25.0^{\circ} \mathrm{C}$. Rev. Col. Cienc. Quím. Farm., Bogotá, v. 32, n. 2, p. 116-136, 2003.

PERLOVICH, G.L.; KURKOV, S.V.; KINCHIN, A.N.; BAUER-BRANDL, A. Thermodynamics of solutions III: Comparison of the solvation of (+)-naproxen with other NSAIDs. Eur. J. Pharm. Biopharm., Amsterdam, v. 57, n. 2, p. 411-420, 2004.

SANGSTER, J. Octanol-water partition coefficients: fundamentals and physical chemistry. Chichester: John Wiley \& Sons, 1997. p. 1-55.

SORENSEN, J.M.; ARTL, W. (1979). Liquid-Liquid Equilibrium Data Collection, Chemistry Data Series, Vol. V, Part I, Frankfurt/Main. apud SANGSTER, J. Octanol-water partition coefficients: fundamentals and physical chemistry. Chichester: John Wiley \& Sons, 1997. p. 1-55.

STEPHENSON, R.; STUART, J.; TABAK, M. (1984). $J$. Chem. Eng. Data, New York, v. 29, p. 287. apud SANGSTER, J. Octanol-Water Partition Coefficients: Fundamentals and Physical Chemistry. Chichester: John Wiley \& Sons, 1997. p. 1-55.

TANFORD, C. Standard states in the thermodynamics of transfer. J. Phys. Chem., Washington, v. 83, n. 13, p. 18021803, 1979. 
VAN NESS, H.C.; ABBOTT, M.M. Classical Thermodynamics of nonelectrolyte solutions. New York: McGraw Hill Book Company, 1982. p. 98-100.

VON ERICHSEN, L. (1952). Brennstoff-Chem., v. 33, p. 166. apud SANGSTER, J. Octanol-water partition coefficients: fundamentals and physical chemistry. Chichester: John Wiley \& Sons, 1997. p. 1-55.
YALKOWSKY, S.H. Solubility and solubilization in aqueous media. New York: American Chemical Society and Oxford University Press, 1999. p. 56-58.

Recebido para publicação em 26 de outubro de 2004. Aceito para publicação em 10 de fevereiro de 2005.

Anexo 1. Análisis de regresión de van’t Hoff usando temperatura armónica de los datos de Dallos y Liszi $\ln X_{2}=a+b\left(\frac{1}{T}-\frac{1}{T_{h m}}\right)+c\left(\frac{1}{T}-\frac{1}{T_{h m}}\right)^{2}$

\begin{tabular}{lcccc}
\hline Sistema & $a$ & $b$ & $c$ & $\mathrm{r}^{2}$ \\
\hline $\mathrm{W}_{(\mathrm{ROH})}$ & -9.69064 & -959.603 & 940164 & 0.9990 \\
$\mathrm{ROH}_{(\mathrm{W})}$ & -1.28705 & -135.134 & 181488 & 0.9972 \\
\hline
\end{tabular}

Anexo 2. Análisis de regresión según el método de Grant et al. de los datos de Dallos y Liszi

$\ln X_{2}=a \frac{1}{T}+b \ln T+c$

\begin{tabular}{lcccc}
\hline Sistema & $a$ & $b$ & $c$ & $\mathrm{r}^{2}$ \\
\hline $\mathrm{W}_{(\mathrm{ROH})}$ & 5064.74 & 19.85920 & -139.877 & 0.9989 \\
$\mathrm{ROH}_{(\mathrm{W})}$ & 1050.41 & 3.90760 & -27.0809 & 0.9972 \\
\hline
\end{tabular}

Anexo 3. Análisis de regresión de van’t Hoff de todos los datos utilizados en este trabajo

$\ln X_{2}=a+b\left(\frac{1}{T}\right)+c\left(\frac{1}{T}\right)^{2}$

(Ec. 25)

\begin{tabular}{llccccc}
\hline Sistema & Autor & $a$ & $b$ & $c$ & $n$ & $\mathrm{r}^{2}$ \\
\hline $\mathrm{W}_{(\mathrm{ROH})}$ & Dallos y Liszi & 3.70511 & -7162.24 & 940164 & 8 & 0.9990 \\
$\mathrm{~W}_{(\mathrm{ROH})}$ & Sorensen y Artl & 0.9577 & -3115.9 & - & 4 & 0.9693 \\
$\mathrm{~W}_{(\mathrm{ROH})}$ & Lebedinskaya et al. & -4.9839 & -1425.1 & - & 4 & 0.9872 \\
$\mathrm{ROH}_{(\mathrm{W})}$ & Dallos y Liszi & 1.13356 & -1332.48 & 181488 & 8 & 0.9972 \\
$\mathrm{ROH}_{(\mathrm{W})}$ & Sorensen y Artl & -3.96928 & 2597.40 & -561376 & 4 & 0.9999 \\
$\mathrm{ROH}_{(\mathrm{W})}$ & Hefter & 9.07324 & -5860.39 & 821358 & 5 & 0.9994 \\
$\mathrm{ROH}_{(\mathrm{W})}$ & Stephenson et al. & -0.3604 & -299.73 & - & 9 & 0.9647 \\
$\mathrm{ROH}_{(\mathrm{W})}$ & Von Erichsen & 1.1156 & -738.89 & - & 10 & 0.9995 \\
\hline
\end{tabular}

Anexo 4. Análisis de regresión por el método de Clausius-Clapeyron de la presión de vapor (atm) del agua y del $n$-octanol $\ln P_{v}=a+b\left(\frac{1}{T}\right)+c\left(\frac{1}{T}\right)^{2}$ (Ec. 26)

\begin{tabular}{lccccc}
\hline Solvente & $a$ & $b$ & $c$ & $n$ & $\mathrm{r}^{2}$ \\
\hline Agua & 13.976 & -5202.9 & - & 100 & 0.9999 \\
n-Octanol & 7.9856 & 1316.18 & 1133329 & 10 & 0.9999 \\
\hline
\end{tabular}

